JOURNAL OF INTEGRAL EQUATIONS AND APPLICATIONS

Volume 2, Number 3, Summer 1990

\title{
PERTURBATION OF LINEAR ABSTRACT VOLTERRA EQUATIONS
}

\author{
C. CORDUNEANU
}

In their pioneering work on Qualitative Theory of Volterra Equations, J. J. Levin and J.A. Nohel have dealt with nonlinear perturbations of Volterra equations of convolution type. Their joint paper [8] on this topic is, likely, the first in the literature dedicated to this subject.

The author [2] has investigated some perturbation problems, in the case of nonconvolution equations, mostly related to the concept of admissibility for integral operators/equations. The role of the resolvent kernel is particularly emphasized in regard to the behavior of solutions, and the Volterra equations provide most of the illustrations. The case of a convolution equation, but not of Volterra type, is also investigated.

In their recent book [6], the authors dedicate a whole chapter to the perturbation theory of integral/integro-differential equations. Further references can be found in [6], especially those of a more recent date.

The aim of this paper is to discuss perturbed equations associated with abstract Volterra equations. We will consider only the case when the unperturbed equation is linear, namely,

$$
x(t)=(L x)(t)+(N x)(t),
$$

or

$$
\dot{x}(t)=(L x)(t)+(N x)(t) .
$$

In both equations (1) and (2), $L$ stands for a linear abstract Volterra operator acting on some function space, while $N$ denotes a nonlinear operator acting on the same or another function space, not necessarily of Volterra type. Of course, when $N$ is also a Volterra (abstract) operator, the equations (1) and (2) are both of Volterra type.

For the definition of Volterra operators and the basic properties of attached equations we refer the reader to our recent book [5], in which these topics are discussed in some detail. See also [11].

Copyright (C)1990 Rocky Mountain Mathematics Consortium 
It is useful to notice that equation (2), with an initial condition of the form

$$
x(0)=x^{0} \in \mathbf{R}^{n},
$$

can be rewritten in the form (1) if we integrate both sides from 0 to some $t>0$ :

$$
x(t)=x(0)+\int_{0}^{t}(L x)(x) d s+\int_{0}^{t}(N x)(s) d s .
$$

As seen in [5], under rather mild assumptions on $L$ (usually, continuity and linearity), one can always write

$$
\int_{0}^{t}(L x)(s) d s=\int_{0}^{t} k(t, s) x(s) d s
$$

where $k(t, s)$ is a measurable kernel, satisfying various conditions, in accordance with the nature of the function space under consideration. Therefore, (4) becomes

$$
x(t)=\int_{0}^{t} k(t, s) x(s) d s+\left(N_{1} x\right)(t),
$$

with

$$
\left(N_{1} x\right)(t)=\int_{0}^{t}(N x)(s) d s+x^{0}
$$

which shows that the perturbed equation (2) can be reduced to an integral equation of classical type, perturbed by a nonlinear term which might involve abstract Volterra operators. Of course, equation (5) is of the form (1).

It is also worth noticing that the perturbation problem may be more adequately investigated in case of the functional-differential equation (2), than in the case of equation (1).

On the other hand, perturbed equations of the form (5) have been investigated by many authors, under various assumptions on the kernel $k(t, s)$ and the nonlinearity $N_{1} x:[\mathbf{1}, \mathbf{2}, \mathbf{3}, \mathbf{7}, \mathbf{9}, \mathbf{1 0}]$. These references contain more data on these kinds of contributions. A difficulty arising in connection with the direct application of available results to equations 
of the form (5) consists in the fact that the properties of the kernel $k(t, s)$ are not easy to describe in terms of the properties of the solution of the linear equation $x=L x+f$.

Let us go back now to equation (1) and assume that we choose the space $C\left([0, T], \mathbf{R}^{n}\right)$ as an underlying space. The theory of the linear equation

$$
x(t)=(L x)(t)+f(t), \quad t \in[0, T],
$$

in which

$$
f \in C\left([0, T], \mathbf{R}^{n}\right)
$$

and $L$ is a Volterra linear operator continuous on $C\left([0, T], \mathbf{R}^{n}\right)$, has been developed in $[\mathbf{5}, \mathbf{1 1}]$. It is shown in these books that $(6)$ has a unique solution in $C\left([0, T], \mathbf{R}^{n}\right)$, for any $f$ satisfying (7), if $L$ is also compact (it is also convenient to assume $(L 0)(t) \equiv 0)$. Hence, the map $f \rightarrow x=U f$ is a bijection of $C\left([0, T], \mathbf{R}^{n}\right)$ onto itself. Since the inverse map $x \rightarrow f$ is obviously continuous, there results the fact that $U$ is also continuous from $C\left([0, T], \mathbf{R}^{n}\right)$ onto itself. Therefore, there exists a positive number $A>0$ such that

$$
\|x\|=\|U f\| \leq A\|f\|, \quad \forall f \in C\left([0, T], \mathbf{R}^{n}\right),
$$

where the norm means the sup-norm.

One usually denotes $R=U-I$ with $I$, the identity mapping of $C\left([0, T], \mathbf{R}^{n}\right)$, which allows us to write the equation $x=U f$ as

$$
x=f+R f, \quad \forall f \in C\left([0, T], \mathbf{R}^{n}\right) .
$$

The operator $R$ is called the resolvent operator corresponding to $L$, and it is obviously a linear continuous operator on $C\left([0, T], \mathbf{R}^{n}\right)$.

Since equation (1) can be written as $(I-L) x=f$, one has $U=$ $(I-L)^{-1}$, whence

$$
R=(I-L)^{-1}-I=L(L-I)^{-1} .
$$

Formula (10) shows that $R$ is also a Volterra operator on $C\left([0, T], \mathbf{R}^{n}\right)$, as well as its compactness. Indeed, it is sufficient to notice that, under 
our conditions, the inverse of a Volterra operator is also of Volterra type (this follows rather easily from the definition).

From the discussion conducted above one derives that both operators $U$ and $R$ are bijections of $C\left([0, T], \mathbf{R}^{n}\right)$ onto itself. This fact has an important implication in regard to equation (1). Namely, (1) can be rewritten in the equivalent form

$$
x(t)=(N x)(t)+(R N x)(t),
$$

where $R N$ stands for the product (superposition) of the operators $N$ and $R$, i.e., $R N x=R(N x), \forall x \in C\left([0, T], \mathbf{R}^{n}\right)$.

Equation (11) can be dealt with by using various fixed point theorems. For instance, due to the boundedness of $R$ on the space $C\left([0, T], \mathbf{R}^{n}\right)$, it suffices to assume that $N$ satisfies a Lipschitz condition

$$
\|N x-N y\| \leq \lambda\|x-y\|, \quad \forall x, y \in C\left([0, T], \mathbf{R}^{n}\right),
$$

where the constant $\lambda$ is small enough (to be precise, $\lambda<(1+|R|)^{-1}$ ). Then the operator

$$
V x=N x+R N x
$$

is a contraction of $C\left([0, T], \mathbf{R}^{n}\right)$, which implies the existence and uniqueness for (11), and hence for $(1)$, in $C\left([0, T], \mathbf{R}^{n}\right)$.

As mentioned above, $N$ need not be of Volterra type. The only conditions on $N$ are to act on $C\left([0, T], \mathbf{R}^{n}\right)$, and to satisfy (12).

Special cases of the result stated above can be obtained for integral equations of the form

$$
x(t)=\int_{0}^{t} k(t, s) x(s) d s+\int_{0}^{T} K(t, s, x(s)) d s,
$$

provided $k(t, s)$ is a continuous kernel for $0 \leq s \leq t \leq T$, or even a measurable kernel which generates a continuous integral operator on $C\left([0, T], \mathbf{R}^{n}\right)$, while the Urysohn operator

$$
x \rightarrow \int_{0}^{T} K(t, s, x(s)) d s
$$


is acting on $C\left([0, T], \mathbf{R}^{n}\right)$, with $K$ satisfying a Lipschitz-type condition of the form

$$
|K(t, s, x)-K(t, x, y)| \leq k_{0}(t, s)|x-y|,
$$

where $k_{0}(t, s) \geq 0$ a.e. on $[0, T] \times[0, T]$ is a measurable function such that

$$
\underset{t}{\operatorname{ess}-s u p} \int_{0}^{T} k_{0}(t, s) d s \leq \lambda=\text { small enough. }
$$

Another standard result for the existence of solutions to equation (11) in $C\left([0, T], \mathbf{R}^{n}\right)$ is obtained by using the Schauder fixed point theorem, instead of the Banach Contraction Principle. In this case, the perturbation $N$ has to be assumed compact. We notice that the product $R N$ will be compact because $R$ is compact, which means that the operator in the right-hand side of (11) is compact.

The following result can be stated:

THEOREM 1. Consider the perturbed equation (1) under the following assumptions:

(a) The Volterra operator $L$ is linear, continuous and compact on the space $C\left([0, T], \mathbf{R}^{n}\right)$, with $(L 0)(t) \equiv 0$.

(b) The operator $N$ is a compact operator on the space $C\left([0, T], \mathbf{R}^{n}\right)$ such that, for any $r>0$,

$$
\Phi(r)=\sup \{\|N x\| ; x \in C,\|x\| \leq r\}
$$

satisfies

$$
\limsup _{r \rightarrow \infty} \frac{\phi(r)}{r}=l
$$

with small enough $l$.

Then equation (1) has at least one solution in $C\left([0, T], \mathbf{R}^{n}\right)$.

ProOF. As mentioned above, it suffices to prove the existence of a solution to equation (11), which can be written as $x(t)=(V x)(t)$, with $V$ given by (13). 
Since $V$ is a compact operator on the space $C\left([0, T], \mathbf{R}^{n}\right)$, it is sufficient to show that it takes a ball of this space, say

$$
B_{r}=\{x ; x \in C,\|x\| \leq r\}
$$

into itself, i.e., $V B_{r} \subset B_{r}$ for some $r>0$.

From (13) and (18) one derives

$$
\|V x\| \leq(1+|R|) \phi(r), \quad x \in B_{r}
$$

where $|R|$ is the norm of $R$ on the space $C\left([0, T], \mathbf{R}^{n}\right)$. Therefore, the inclusion $V B_{r} \subset B_{r}$ is assured by the inequality

$$
\phi(r) \leq(1+|R|)^{-1} r
$$

if this inequality has at least one positive solution.

But condition (19) guarantees the existence of a sufficiently large $r>0$ such that $(21)$ is satisfied, as soon as we assume

$$
\limsup _{r \rightarrow \infty} \frac{\phi(r)}{r}=l<(1+|R|)^{-1}
$$

The Schauder fixed point theorem applied to the operator $V$, in the space $C\left([0, T], \mathbf{R}^{n}\right)$, leads to the existence of at least one solution of equation (1) in the ball $B_{r}$, with $r$ satisfying (21). $\square$

From Theorem 1 one can obtain as special cases various results regarding perturbed integral equations of Volterra type such as

$$
x(t)=\int_{0}^{t} k(t, s) x(s) d s+\int_{0}^{t} K(t, s, x(s)) d s,
$$

in which $k(t, s)$ is a continuous kernel for $0 \leq s \leq t \leq T$. It remains to impose adequate conditions on $K$ such that the nonlinear part in (23) represents a compact operator on $C\left([0, T], \mathbf{R}^{n}\right)$. Such conditions can be easily obtained if we assume, for instance, that $K(t, s, x)$ is continuous for $0 \leq s \leq t \leq T,|x| \leq M, M>0$. But continuity is not necessary at all to assure the fact $K: C \rightarrow C$. It is sufficient to deal with a $K(t, s, x)$ measurable in $(t, s)$ and continuous in $x$ (Carathéodory condition), such 
that $K(t, s, x)$ is essentially bounded on any set $0 \leq s \leq t \leq T,|x| \leq r$. Moreover, to secure compactness of the integral operator generated by $K(t, s, x)$, one can assume

$$
\lim _{t \rightarrow t_{0}} \int_{0}^{t}\left|K(t, s, x(s))-K\left(t_{0}, s, x(s)\right)\right| d s=0,
$$

uniformly with respect to $x \in B_{r} \subset C$, for any $r>0$.

In the remaining part of this paper we shall deal with perturbed equations of the form (2), which can be brought to the form (5), under adequate conditions. As shown in our book [5], the linear equation associated with (2),

$$
\dot{x}(t)=(L x)(t)+f(t),
$$

under initial condition (3), has a unique solution on $[0, T]$ which is absolutely continuous and satisfies the equation a.e., provided $L$ is a linear continuous operator on $L^{2}\left([0, T], \mathbf{R}^{n}\right)$ of Volterra type and $f$ belongs to the same space.

Since the solution of the problem (25), (3) can be expressed by means of the variation of parameters formula

$$
x(t)=X(t, 0) x^{0}+\int_{0}^{t} X(t, s) f(s) d s,
$$

with $X(t, s)$ constructed as shown in [5], there results that the problem $(2),(3)$ can be reduced to the functional integral equation

$$
x(t)=X(t, 0) x^{0}+\int_{0}^{t} X(t, s)(N x)(s) d s .
$$

In [5], we have used this method of transformation of the differential problem into an integral one, mentioning some properties of the kernel $X(t, s)$. To be more specific, $X(t, s)$ is defined by the formula

$$
X(t, s)=I+\int_{s}^{t} \tilde{k}(t, u) d u
$$

where $\tilde{k}$ stands for the resolvent kernel associated to the Volterra kernel $k(t, s)$ from (5). 
From (26) it follows that the operator

$$
f \rightarrow \int_{0}^{t} X(t, s) f(s) d s
$$

which is defined for any $f \in L^{2}\left([0, T], \mathbf{R}^{n}\right)$, takes its values in the space of absolutely continuous functions on $[0, T]$. Actually, the operator (29) is completely continuous from $L^{2}\left([0, T), \mathbf{R}^{n}\right)$ into $C\left([0, T], \mathbf{R}^{n}\right)$, because when $f$ belongs to a bounded set in $L^{2}\left([0, T], \mathbf{R}^{n}\right), \dot{x}$ from $(25)$ also belongs to a bounded set of the same space.

The above mentioned properties of the operator (29) allow us to deal with the functional-integral equation in the space $C\left([0, T], \mathbf{R}^{n}\right)$. It is adequate to assume that the operator $N$ is acting from the space $C\left([0, T], \mathbf{R}^{n}\right)$ into $L^{2}\left([0, T], \mathbf{R}^{n}\right)$. If we assume $N: C \rightarrow C$, then it is obvious that a result similar to Theorem 1 can be obtained for the problem (2), (3).

THEOREM 2. Consider the problem (2), (3), under the following hypotheses.

(a) The operator $L$ is linear, continuous, and of Volterra type on the space $L^{2}\left([0, T], \mathbf{R}^{n}\right)$.

(b) $N$ is an operator taking the space $C\left([0, T], \mathbf{R}^{n}\right)$ into the space $L^{2}\left([0, T], \mathbf{R}^{n}\right)$, such that

$$
|N x-N y|_{L^{2}} \leq \lambda|| x-y||,
$$

where $\lambda$ is a sufficiently small constant.

Then there exists a unique solution in $C\left([0, T], \mathbf{R}^{n}\right)$ of the problem (2), (3), which is absolutely continuous.

The proof is straightforward and is based on Banach contraction mapping in the space $C\left([0, T], \mathbf{R}^{n}\right)$, applied to the operator appearing in the right-hand side of equation (27). We omit the details.

REMARK. If condition (a) of Theorem 2 is verified and condition (b) is replaced by the continuity of $N$ from $C\left([0, T], \mathbf{R}^{n}\right)$ into $L^{2}\left([0, T], \mathbf{R}^{n}\right)$, plus a growth condition for $N$ similar to (22), then the existence of a solution to the problem (2), (3) is guaranteed. 
Indeed, the Schauder fixed point theorem is applicable to the operator appearing in the right-hand side of equation (27), which takes a ball $B_{r}$ of $C\left([0, T], \mathbf{R}^{n}\right)$ into itself. The compactness of this operator guarantees the existence of a fixed point, which is a solution of (2), (3).

We notice that these results can be easily applied to integrodifferential equations such as

$$
\dot{x}(t)=\int_{0}^{a(t)} k(t, s) x(s) d s+\int_{0}^{b(t)} K(t, s, x(s)) d s,
$$

where $0 \leq a(t), b(t) \leq t$ on $[0, T]$ are given functions with convenient properties.

\section{REFERENCES}

1. G. Bantas, On the asymptotic behavior in the theory of Volterra integrofunctional equations, Period. Math. Hungar. 5 (1974), 323-332.

2. C. Corduneanu, Some perturbation problems in the theory of integral equations, Math. Systems Theory 1 (1967), 143-155.

3. Integral equations and stability of feedback systems, Academic Press, New York, 1973.

4. - Some global problems for Volterra functional-differential equations, in Volterra Integro-differential Equations in Banach Spaces and Applications (G. DaPrato and M. Iannelli, eds.), Longman, London, 1989, 90-100.

5. - Integral equations and applications, Cambridge Univ. Press, New York, 1990.

6. G. Gripenberg, S.O. Londen, and O. Staffans, Volterra integral and functional equations, Cambridge Univ. Press, New York, 1990.

7. J.J. Levin, Nonlinearly perturbed Volterra equations, Tôhoku Math. J. (2) 32 (1980), 317-335.

8. J.J. Levin and J.A. Nohel, Perturbations of a nonlinear Volterra equation, Michigan Math. J. 12 (1965), 431-447.

9. R.K. Miller, On the linearization of Volterra integral equations, J. Math. Anal. Appl. 23 (1968), 198-208.

10. - Admissibility and nonlinear Volterra integral equations, Proc. Amer. Math. Soc. 25 (1970), 65-71.

11. L. Neustadt, Optimization: A theory of necessary conditions, Princeton Univ. Press, Princeton, N.J., 1976.

Mathematics Department, University of Texas at Arlington, Arlington, TX 76019 